Article

\title{
Dynamically Tunable Plasmon-Induced Transparency Based on Radiative-Radiative-Coupling in a Terahertz Metal-Graphene Metamaterial
}

\author{
Guanqi Wang ${ }^{1}{ }^{1}$, Xianbin Zhang ${ }^{1, *}$, Lei Zhang ${ }^{2}$ and Xuyan Wei ${ }^{1}$ \\ 1 Department of Applied Physics, Xi'an University of Technology, Xi'an 710048, China; \\ wangguanqi1978@sina.com (G.W.); XuYan_Wei117@163.com (X.W.) \\ 2 Key Laboratory for Physical Electronics and Devices of the Ministry of Education \& Shaanxi Key Lab of \\ Information Photonic Technique, Xi'an Jiaotong University, Xi'an 710049, China; eiezhanglei@xjtu.edu.cn \\ * Correspondence: gqwang@stu.xaut.edu.cn; Tel.: +86-29-8206-6351
}

Received: 29 January 2019; Accepted: 7 March 2019; Published: 13 March 2019

\begin{abstract}
New technologies and materials with superior characteristics impel great development of functional devices in the terahertz field. The dynamically tunable plasmon-induced transparency (PIT) based on radiative-radiative-coupling in terahertz hybrid metal-graphene metamaterial is numerically investigated in this paper. For the active manipulation of the PIT device, the single-layer graphene is integrated into the proposed structure consisting of the split-ring-resonator (SRR) and the closed-ring-resonator (CRR). Dynamically adjusting Fermi energy in graphene leads to modulation of the PIT window, allowing for the active control of the group delay. From the simulated electrical field distributions and effective circuit model to analyze, the transmission spectrum modulation can be attributed to the altering in the energy loss of the dark mode resonator through the conduction effect of the graphene layer. Our work offers theoretical references for the development of slow light terahertz devices in the future.
\end{abstract}

Keywords: graphene; metamaterial; terahertz; plasmon-induced transparency

\section{Introduction}

Electromagnetically induced transparency (EIT) is a very important phenomenon in physics, which is the result of quantum destructive interference. Electromagnetically induced transparency has important application in slow light effect, information storage and non-linear effect [1-3]. However, the implementation of EIT effect in the original atomic system often requires harsh conditions such as ultra-low operating temperatures and strong light pumping, which makes EIT effect restrictive in practical applications [4]. The emergence of metamaterials lays new roads to modulate light in the sub-wavelength range. Metamaterial-based plasmon-induced transparency (PIT) is an optical analogue of EIT, which has attracted much attention owing to its flexible design and easy implementation $[5,6]$. The PIT metamaterials introduce the quantum effect into the classic optics field and show unique potentials in developing many new types of compact devices in the terahertz $(\mathrm{THz})$ range.

In general, there are usually two ways for realizing PIT: radiative-dark-coupling [7-10] and radiative-radiative-coupling [11-14]. In order to speed-up practical application, dynamically tunable PIT metamaterials have been extensively studied.

In recent years, much research has been conducted on achieving dynamically tunable PIT windows by integrating optically active materials with metamaterials, such as non-linear materials, liquid crystals, and semiconductors [15-17]. Among these active materials, graphene, a novel two-dimensional material, has built a wonderful platform to achieve dynamically tunable $\mathrm{THz}$ 
devices attributable to its excellent electronic and optical properties $[18,19]$. On the one hand, flexible modulation in the conductivity of graphene is induced by changing Fermi energy via electrostatic gating, which is easy to operate in experiments. On the other hand, the modulation speed of the graphene metamaterial is in the picosecond order due to the ultrafast response of graphene to terahertz waves. Therefore, various graphene-based metamaterials have been designed to produce controllable PIT [20-25]. However, the surface conductivity of patterning graphene with resonance effects is difficult to adjust, which limits it practical application.

Here, we present an active modulation of PIT terahertz metamaterial by combining a graphene layer into an Al-based metamaterial system consisting of the split-ring-resonator (SRR) and the closed-ring-resonator (CRR). When the Fermi energy of graphene changes, the modulation depth of the PIT window can be dynamically tuned on a large scale, while the frequency of the PIT peak remains unchanged. According to the simulated electrical field distributions and effective circuit model, we elaborated the physical mechanism of active manipulation of PIT metamaterials owing to the conduction effect of the metallic graphene layer. Besides, the slow light effect of the proposed metamaterial is significant, and the group delay time can be adjusted in a wide range of picoseconds. Our work paves the way for new perspectives for the ultra-fast active control of terahertz devices.

\section{Proposed Structure and Methods}

The unit cell of the proposed metal-graphene metamaterial consisting of a split-ring-resonator and a closed-ring-resonator arranged in order, as is illustrated in Figure 1 [26]. The meta-atoms structures are assembled on the top of a semi-infinite silicon (Si) substrate with permittivity $\varepsilon_{s i}=11.7$. The constructed unit cells are made of aluminum with a thickness of $0.2 \mu \mathrm{m}$. In this research, the dielectric constant of the $\mathrm{Al}$ at $\mathrm{THz}$ frequencies can be expressed as the Drude model [27]:

$$
\varepsilon=\varepsilon_{\infty}-\frac{\omega_{p}^{2}}{\omega^{2}+i \omega \gamma}
$$

where the high-frequency dielectric constant is $\varepsilon_{\infty}=1$, the plasma frequency is $\omega_{p}=11.9 \times 10^{4} \mathrm{~cm}^{-1}$, and the damping constant is $\gamma=6.6 \times 10^{2} \mathrm{~cm}^{-1}$.

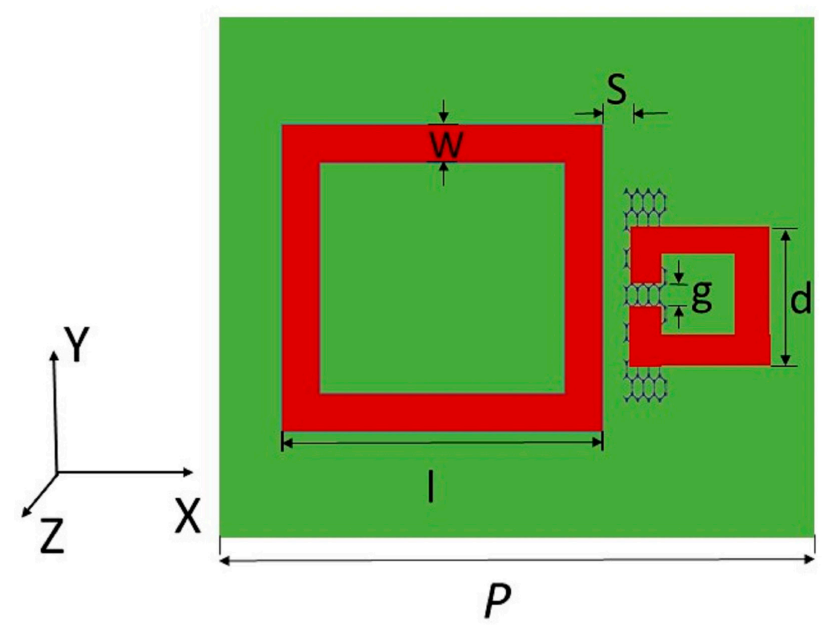

Figure 1. Schematic of a unit cell with geometrical parameters: $1=70 \mu \mathrm{m}, \mathrm{d}=31 \mu \mathrm{m}, \mathrm{W}=5 \mu \mathrm{m}$, $\mathrm{S}=5 \mu \mathrm{m}, \mathrm{g}=5 \mu \mathrm{m}, \mathrm{P}=120 \mu \mathrm{m}$.

In order to achieve the dynamically manipulating radiative-radiative-coupling in PIT devices, a two-dimensional graphene flat sheet is deposited under the split gap of SRR.

For exploring the underlying physics in the designed structure, simulated calculations based on the finite difference time-domain (FDTD) method were carried out. During the electromagnetic wave 
simulation, the linearly polarized terahertz wave was normally incident to the PIT structure along the z-direction, with the electric field $\vec{E}$ polarized along the y-direction, and the magnetic field $\vec{H}$ along the $x$-direction.

\section{Conductivity of Graphene}

As is known, graphene is a tunable optical 2D material whose optical properties can be described by its complex surface conductivity. Graphene conductivity consists of the intra-band and inter-band transition processes. It can be calculated according to the Kubo formula [19,28]:

$$
\begin{gathered}
\sigma=\sigma_{r}+i \sigma_{i}=\sigma_{\text {intra }}+\sigma_{\text {inter }}, \\
\sigma_{\text {intra }}=\frac{i e^{2} k_{B} T}{\pi \hbar^{2}\left(\omega+i \tau^{-1}\right)}\left[\frac{E_{F}}{k_{B} T}+2 \ln \left(e^{-\frac{E_{F}}{k_{B} T}}+1\right)\right], \\
\text { and } \sigma_{\text {inter }}=\frac{e^{2}}{4 \hbar}\left[\theta\left(\omega-2 E_{F} / \hbar\right)-\frac{i}{2 \pi} \ln \frac{\left(\omega+2 E_{F} / \hbar\right)^{2}}{\left(\omega-2 E_{F} / \hbar\right)^{2}}\right],
\end{gathered}
$$

where $T, e, \hbar$, and $k_{B}$ are the operation temperature, the elementary charge, the reduced Planck constant, and the constant of Boltzmann, respectively. $\omega, E_{F}$ and $\tau$ stand for the photon frequency, Fermi energy in graphene and carrier relaxation time, respectively. The step function $\theta(\omega)$ express the condition for inter-band transition.

From the far infrared to the terahertz range, the inter-band process is negligible due to the suppression of inter-band transitions because of Pauli state blocking, and the intra-band transition dominates when $\hbar \omega<2 E_{F}$. If the conditions $E_{F} \gg k_{B} T$ and $E_{F} \gg \hbar \omega$ are satisfied, the surface conductivity can be further simplified to a Drude-like form [29]:

$$
\sigma_{\text {gra }}=\frac{e^{2} E_{F}}{\pi \hbar^{2}} \frac{i}{\omega+i / \tau} .
$$

In summary, the connection between the Fermi velocity $v_{F}$, the carrier mobility $u$, carrier relaxation time $\tau$, and the Fermi energy $E_{F}$ can be described as $\tau=u E_{F} /\left(e v_{F}^{2}\right)$, which indicates that the carrier mobility has an important influence on the ohmic loss of the graphene. In the simulation, we employed $v_{F}=1.1 \times 10^{6} \mathrm{~m} / \mathrm{s}$ and $u=3000 \mathrm{~m}^{2} /(V \cdot s)$, which was achieved in the experiment [30].

It can be inferred from the Equation (5) that the graphene surface conductivity can be flexibly tuned by altering Fermi energy. As a result, the frequency-dependent graphene conductivity under different Fermi energy was calculated based on the Drude-like model and shown in Figure 2. It was noteworthy that both the real part and imaginary part of graphene conductivity rapidly decreased as the Fermi energy decreased. When the graphene Fermi energy gradually approached the Dirac point, the density of states available for transitions decreased, resulting in a decrease in graphene conductivity. Moreover, it was observed that the conductivity of the imaginary part of graphene was positive, then the real part of the graphene permittivity constant was negative, which is ruled by $\varepsilon=i \sigma / \omega$. Therefore, graphene exhibits metallic character and thus can support plasmonic effect in the terahertz region [19]. Relative to other thin metal layers and metal surfaces, the significant advantage of graphene is the capability of dynamically tuning the conductivity by electrical voltage or chemical doping. This conductivity-adjustable metal analogue can also be used as a key material to control the opening and closing of the gaps in the metamaterial structure, providing more possibilities for the tunable terahertz metamaterials [31]. In other words, there are two aspects to the application of graphene electrodynamic properties, one is as plasma material, the other is as conductive material. 


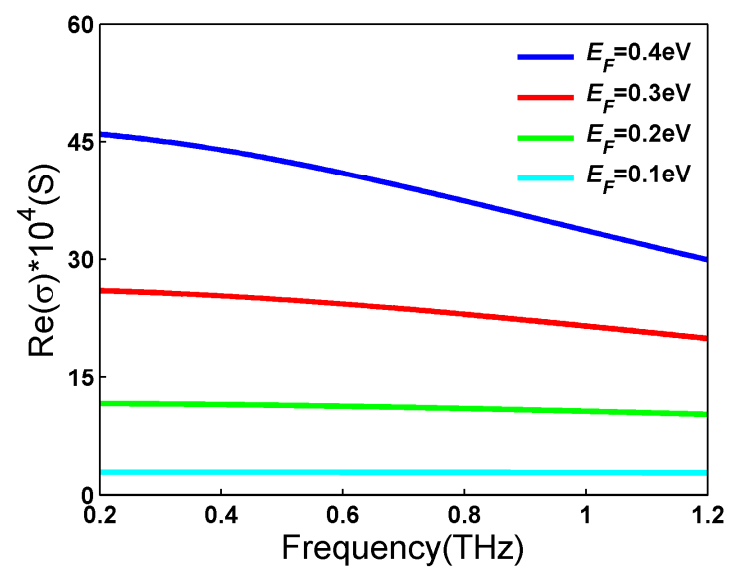

(a)

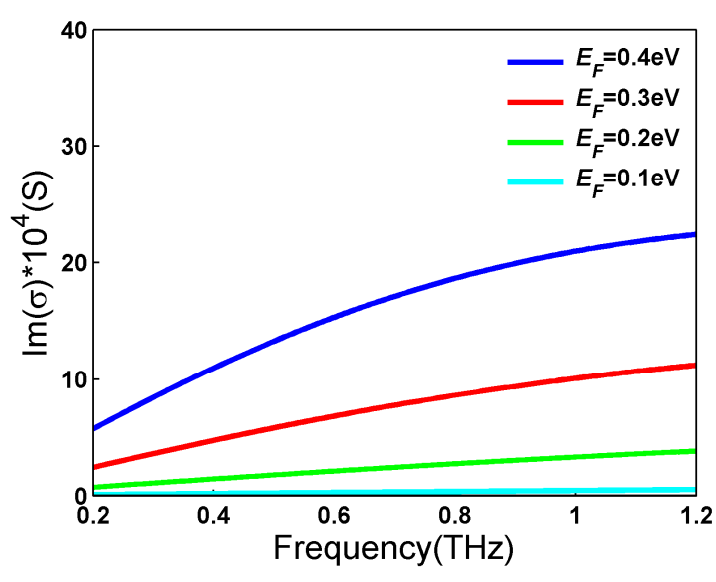

(b)

Figure 2. The frequency dependent graphene conductivity under different Fermi energy: (a) real and (b) imaginary parts. The Fermi energy varied from $0.1 \mathrm{eV}$ to $0.4 \mathrm{eV}$.

\section{General Modulation Properties}

To demonstrate the PIT effect in the designed graphene-based metamaterial, three structures were simulated, including an SRR array, a CRR array, and a combined SRR and CRR array, and the transmission spectra are presented in Figure 3. The CRRs exhibited a broad resonance absorption valley, with a center frequency of $0.56 \mathrm{THz}$ and an amplitude of 0.01 . However, the SRRs exhibited a narrow resonance absorption valley, with a center frequency of $0.60 \mathrm{THz}$ and an amplitude of 0.13 . Hence, there was an extremely finite frequency detuning between the CRRs and SRRs. When forming a one-unit cell, including the CRRs and SRRs, an obvious transparency window was exhibited at $0.58 \mathrm{THz}$ between the resonance frequencies of the CRRs and SRRs, namely, the PIT phenomenon in the metamaterial.

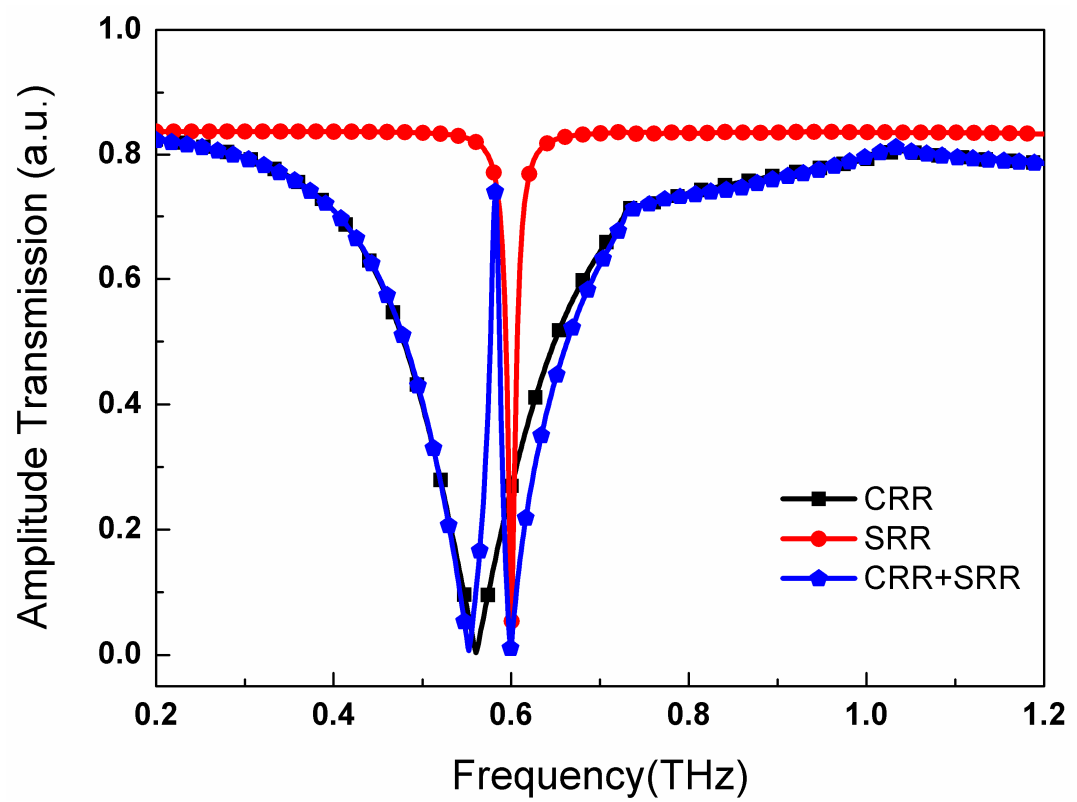

Figure 3. The simulated amplitude transmission spectra of the closed-ring-resonator (CRR), the split-ring-resonator (SRR), and both of them.

Both CRRs and SRRs can be independently excited via a terahertz normal incidence with polarization in the y-direction. Symmetric CRRs can intensely electromagnetically couple with the incident terahertz wave because of the even order eigenmode. With an external terahertz radiation 
field, CRRs will exhibit electric dipole oscillation, i.e., the lowest even eigenmode. This resonance mode exhibits a wide full-width of half maximum (FWHM) and low-quality factor. On the other hand, the asymmetric SRRs can be excited to form an LC oscillation eigenmode, and the surface current is circulation distributions. This resonance mode exhibits a narrower FWHM and high-quality factor due to weak external electric field coupling, as shown in Figure 3. Hence, CRRs behave as the bright mode resonator and SRRs serve as the dark mode resonator.

Analyzing from Faraday's law of electromagnetic induction, there is a strong interaction between the dipole surface current in the CRR and the circulation current in the SRR. Based on the coupling effect, the reciprocating coupling of the electromagnetic field between the CRR and the SRR, but with a $\pi$ phase difference, leads to a classical destructive interference [32].

In order to achieve active control of the PIT device, the single-layer graphene was integrated under the split gap of the SRR, as depicted in Figure 1. Surprisingly, the coupling strength between the bright and dark mode the resonator were fully controlled via altering the Fermi energy of the graphene layer. Figure 4 shows the active amplitude transmission spectra of the proposed system by tuning the Fermi energy $E_{F}$ in graphene. The amplitude undergoes an on-to-off modulation behavior of the PIT window at $0.58 \mathrm{THz}$ without affecting adjacent frequency spectra. To quantitatively describe the extinction amplitude induced by the single-layer graphene, here we introduce the modulation depth in the transmission as:

$$
M=\frac{\left|T_{g}-T_{0}\right|}{T_{0}} \times 100 \%
$$

where, the transmissions at the transparency peak of the PIT without and with the graphene layer are $T_{0}$ and $T_{g}$, respectively. Initial stage $E_{F}$ was $0.1 \mathrm{eV}$, the modulation depth in the transmission was $M_{0.1}=55 \%$. When $E_{F}$ increased to $0.2 \mathrm{eV}$, the modulation depth rose to $M_{0.2}=66.4 \%$. Finally, when $E_{F}$ added up to $0.4 \mathrm{eV}$, the modulation depth went to $M_{0.4}=91.6 \%$. There is a single resonance at around $0.58 \mathrm{THz}$ that can be observed. Thus, we have achieved a whole manipulation in the resonance strength of the PIT device. In addition, the perfect manipulation of the PIT only needs an $E_{F}$ of $0.4 \mathrm{eV}$, which is easy to implement experimentally [33]. These means that the modulation of the PIT amplitude can be realized by applying a gate voltage, which is more in line with actual needs and can be well applied to the absorption bleaching of actual terahertz sources.

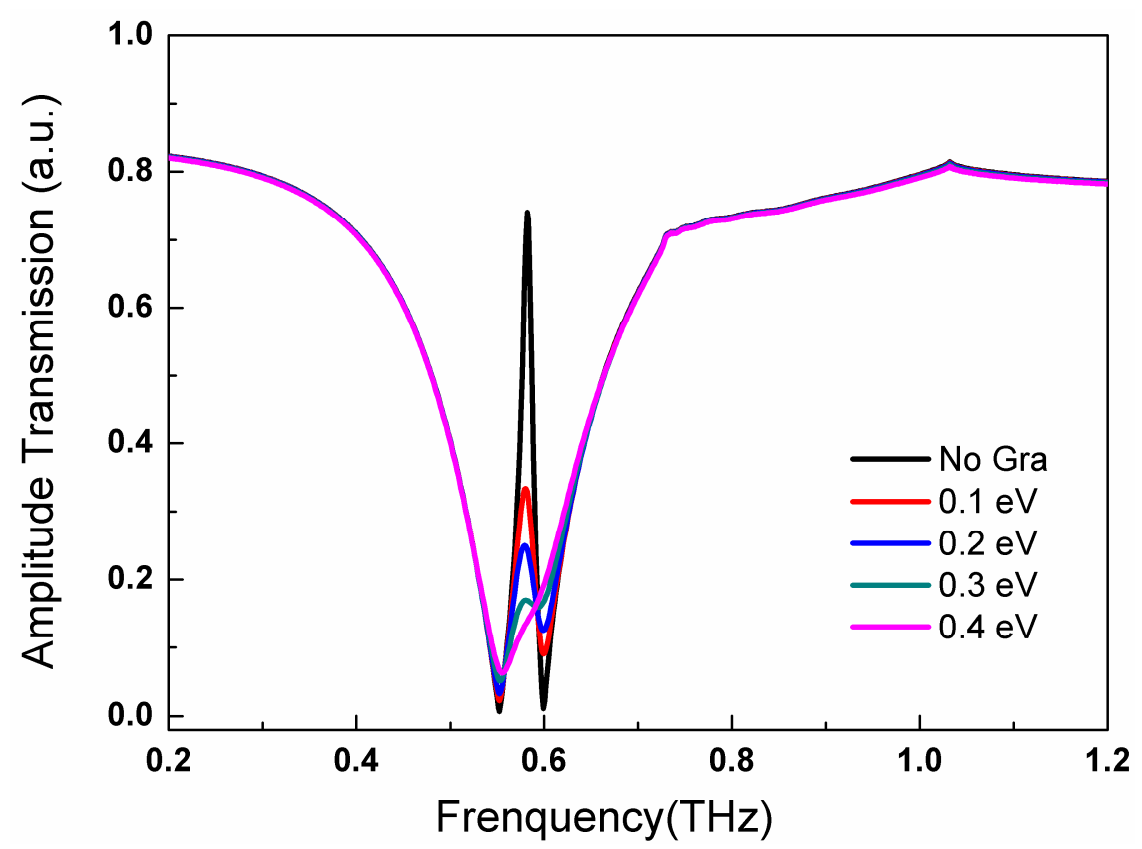

Figure 4. The simulated amplitude transmission spectra of the proposed metamaterials at different Fermi energy of single-layer graphene. 
To reveal the modulation mechanism of the proposed PIT, the distributions of the electric field at the PIT resonance frequency were investigated. In Figure 5, we show the near-field distributions of the total electric field intensity $E=\sqrt{\left|E_{x}\right|^{2}+\left|E_{y}\right|^{2}+\left|E_{z}\right|^{2}}$ at $\mathrm{f}=0.58 \mathrm{THz}$ for the cases without graphene and for $E_{F}=0.1 \mathrm{eV}$ and $E_{F}=0.4 \mathrm{eV}$. Without graphene layer in the structure unit, the LC dark mode and the electric dipole oscillation bright mode interfered destructively with each other, which reflected the strong distribution of the electric field in the split gap of the dark SRR. In the destructive interference process, the electric field enhancement transfers from the bright mode to the dark mode resonator, similar to the quantum EIT effect. Because the split gap of an SRR is equivalent to a capacitor, the opposite charges accumulate at its two ends. When the graphene layer is integrated into the proposed structure, the opposite charges are recombined through the conductive graphene channels, which can attenuate the effect of capacitance in the split gap of the SRR. Hence, the electric field enhancement in the split gap is weakened, as shown in Figure 5b. The surface conductivity of graphene can be dynamically adjusted by changing the Fermi energy, which means that the increase in the Fermi energy leads to a reduction in the capacitive effect in the gap of the dark SRR. When the Fermi energy increases to $0.4 \mathrm{eV}$, there is significant enhancement of the graphene recombining effect with larger conductivity, and the capacitance effect is significantly weakened. Therefore, the SRR exhibited too little capacitance to sustain the LC resonance mode, and the local field enhancement on SRR almost disappears, as shown in Figure 5c. Through the metal-like conductive graphene, the capacitance effect in the split gap of the SRR was modulated. However, the electric field distribution on the dark mode CRR was only slightly changed with the change in the Fermi energy, which is attributed to its small quality factor and gentle resonance spectrum. It can be determined that dynamically-tunable PIT based on radiative-radiative-coupling is possible because of the modulation of graphene surface conductivity by adjusting its Fermi energy.

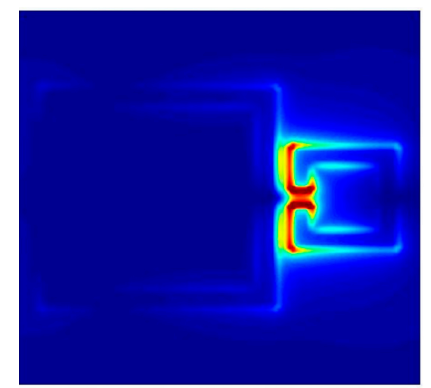

(a)

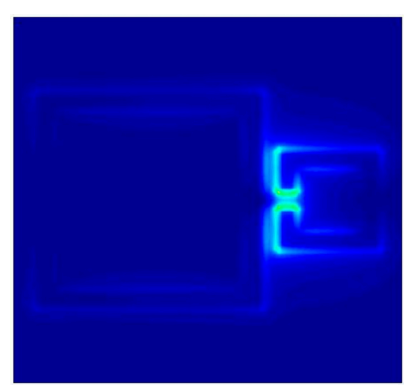

(b)

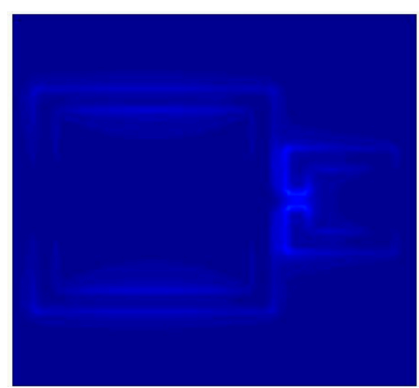

(c)

Figure 5. The electric field distributions of the plasmon-induced transparency (PIT) metamaterials at $0.58 \mathrm{THz}$ for the cases (a) without graphene; (b) $E_{F}=0.1 \mathrm{eV}$; and (c) $E_{F}=0.4 \mathrm{eV}$.

\section{Effective Circuit Model Explanation}

For a better understanding of the radiative-radiative-coupling in the PIT effect, we used the equivalent RLC circuit model [34], as shown in Figure 6. The left and right loops represent the bright mode and dark mode resonances, respectively. Without the single-layer graphene, which is shown in Figure $6 a$, the loop on the left consisting of $R_{1}, L_{1}, C_{1}$ and the loop on right consisting of $R_{2}, L_{2}$, $\mathrm{C}_{2}$ forms a coupled resonant circuit having resonance at $\mathrm{f}=0.58 \mathrm{THz}$. The coupling effect between the dark and bright modes is described by $C_{3}$, and $C_{2}$ represents the capacitance effect in the split gap of the dark SRR. $V_{1}$ and $V_{2}$ represent the terahertz excitation field in the bright and dark modes, respectively. Based on the conduction effect of metallic graphene, the single-layer graphene is seen as a resistance $R_{g}$ that parallels with $\mathrm{C}_{2}$, as shown in Figure 6b. As for the LC resonance, $R_{g}$ will weaken the dark mode capacitor $C_{2}$ and enhance the energy loss in the SRR. With increasing $E_{F}$, the energy losses in the dark mode will further increase, caused by the enhancement of the conduction effect of metallic graphene, which eventually leads to the disappearance of the PIT effect. 


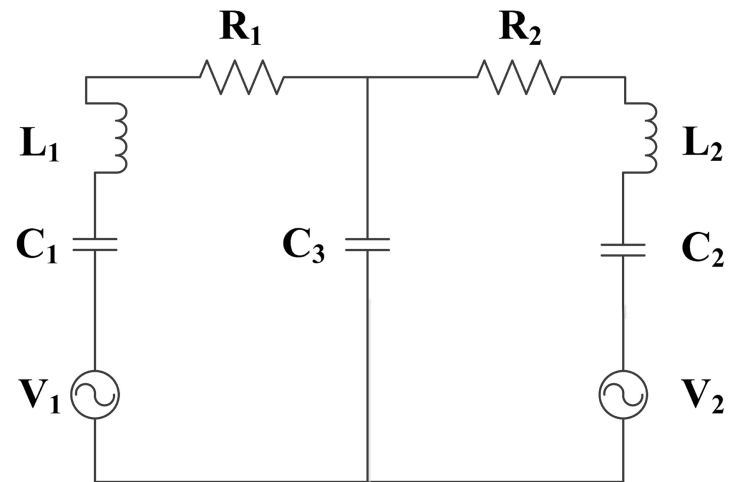

(a)

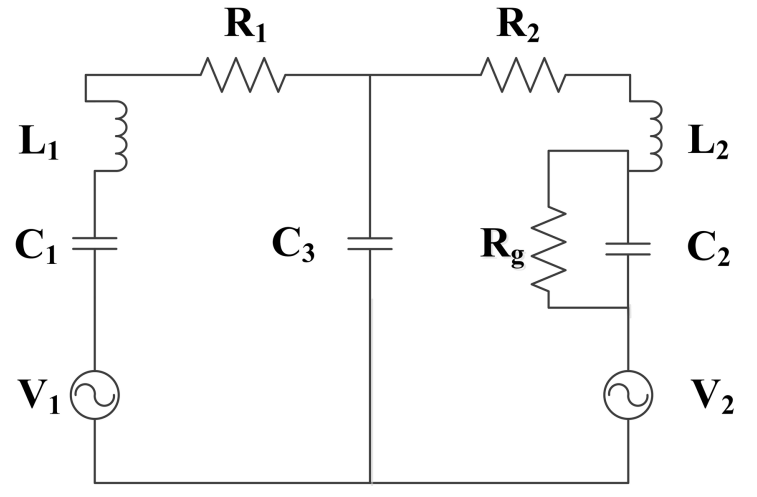

(b)

Figure 6. Effective circuit model of PIT without (a) and with (b) the graphene layer.

For achieving strong coupling, the bright mode and dark mode resonators must have extremely near resonance frequency. Based on the expression $\omega_{S R R} \propto 1 / \sqrt{L_{2} C_{2}}$, the introduction of $R_{g}$ can alter the dark mode resonance frequency. The relative high conductivity corresponds to a relative low $R_{g}$, which will further decrease the quality factor and weaken the strength of coupling. Hence, we can achieve the manipulation of PIT by controlling the strength of radiative-radiative coupling.

\section{Slow Light Effect}

The PIT effect has been well proved to apparently slow down the speed of light and have potential applications in the field of optical signal processing [35]. The actively tuning amplitude transmission spectra in Figure 3 indicate that the proposed PIT system can realize the dynamical modulation of the group velocity of the terahertz wave. This is mainly reflected in the group velocity index, because the greater the group velocity index, the more severe the group velocity delay. The group velocity refractive index $n_{g}$ in the transparency window can be expressed as [23]:

$$
n_{g}=n+\omega \frac{d n}{d \omega}
$$

where $n$ is the effective refractive index for the PIT metamaterial. In this paper, we introduce the group delay $t_{g}$ to depict the slow light capability, which is expressed as [21]:

$$
t_{g}=\frac{d \psi}{d \omega}
$$

where $\psi$ is the transmission phase shift. The group delay $t_{g}$ is more suitable for distinguishing the slow light capability of the device than the group refractive index $n_{g}$, because the solution discards the effective thickness of the metamaterial, which is complicated to define due to the influence of the substrate.

As shown in Figure 7, we calculated the transmission group delay of the PIT metamaterials for different Fermi energy. Without the single-layer graphene, the PIT metamaterial shows the largest group delay $t_{g}=19.2 \mathrm{ps}$ at the EIT peak, which corresponds to a group delay of a $5.76 \mathrm{~mm}$ distance of the vacuum propagation. By integrating the single-layer graphene into the metal unit cell, as $E_{F}$ increases, the maximum value of $t_{g}$ gradually decreases, which means the gradual loss of the slow light capability of the metamaterial. When $E_{F}=0.4 \mathrm{eV}$, the slow light characteristic of PIT metamaterial almost disappears. The flexible controlled group delay over large timescales in the proposed metamaterial is comparable to previous reports [21,36,37], which is due to the dark mode of high-quality factors in SRRs. Therefore, the proposed PIT metamaterial has the ability to actively regulate slow light, which exhibits great promise in developing optical communication and optical storage devices. 


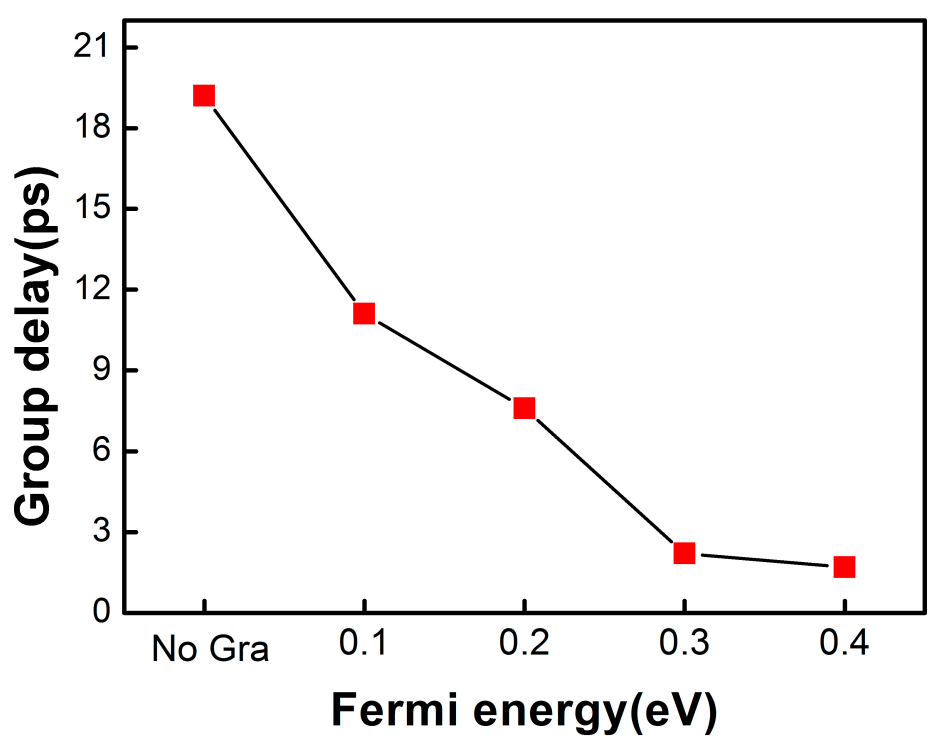

Figure 7. The maximum group delay with the PIT window versus Fermi energy of graphene.

\section{Conclusions}

In summary, the single-layer graphene as the active medium of dynamic metamaterials to effectively modulate the PIT effect was investigated. The transparency peak resulted from the radiative-radiative coupling between SRRs and CRRs, in which the capacitive effect of the gap in SRRs plays a significant role. Most importantly, by adjusting the graphene Fermi energy instead of the geometrical parameters, the PIT window can be actively modulated without affecting adjacent frequency spectra, and the maximum modulation depth of $91.6 \%$ could be obtained. The electric field further revealed that the underlying physics lies in the conduction effect of metallic graphene. The effective circuit model was applied to describe the PIT response to the presented structure. In addition, the maximum value of the group delay within the PIT window declined from 19.2 to $1.7 \mathrm{ps}$ as the Fermi energy increased. This feature may have great potential in the development of slow light devices, which helps to stimulate the development of terahertz communication. Our work provides a new perspective for the fusion of graphene and metamaterials in the terahertz region.

Author Contributions: G.W. conceived the idea; G.W. and X.W. performed the numerical simulations; X.Z. and L.Z. provided the guidelines for the paper; G.W., X.Z., and L.Z. participated in the discussions and writing the article.

Funding: Our work was funded by the National Natural Science Foundation of China (Nos. 61377036 and 60971015).

Acknowledgments: We are grateful to Bing Wei (Xidian University) for the beneficial discussion on the finite difference time-domain method in the THz range.

Conflicts of Interest: The authors declare not any conflict of interest.

\section{References}

1. Fleischhauer, M.; Imamoglu, A.; Marangos, J.P. Electromagnetically induced transparency: Optics in coherent media. Rev. Mod. Phys. 2005, 77, 633-673. [CrossRef]

2. Hau, L.V.; Harris, S.E.; Dutton, Z.; Behroozi, C.H. Light speed reduction to 17 metres per second in an ultracold atomic gas. Nature 1999, 397, 594-598. [CrossRef]

3. Zhang, Y.P.; Brown, A.W.; Xiao, M. Opening four-wave mixing and six-wave mixing channels via dual electromagnetically induced transparency windows. Phys. Rev. Lett. 2007, 99, 123603. [CrossRef] [PubMed]

4. Zhu, Y.F.; Lin, J. Sub-Doppler light amplification in a coherently pumped atomic system. Phys. Rev. A. 1996, 53, 1767-1774. [CrossRef] 
5. Zhang, S.; Genov, D.A.; Wang, Y.; Liu, M.; Zhang, X. Plasmon-induced transparency in metamaterials. Phys. Rev. Lett. 2008, 101, 047401. [CrossRef]

6. Lin, X.Q.; Yu, J.W.; Chen, Z.; Zhang, P.C.; Li, P.F. Analogue of electromagnetically induced transparency with modulatable transmission. Electron. Lett. 2015, 51, 1132-1133. [CrossRef]

7. Yang, Y.M.; Kravchenko, I.I.; Briggs, D.P.; Valentine, J. All-dielectric metasurface analogue of electromagnetically induced transparency. Nat. Commun. 2014, 5, 5753. [CrossRef]

8. Singh, R.; Rockstuhl, C.; Lederer, F.; Zhang, W.L. Coupling between a dark and a bright eigenmode in a terahertz metamaterial. Phys. Rev. B. 2009, 79, 085111. [CrossRef]

9. Devi, K.M.; Sarma, A.K.; Chowdhury, D.R.; Kumar, G. Plasmon induced transparency effect through alternately coupled resonators in terahertz metamaterial. Opt. Express 2017, 25, 10484-10493. [CrossRef] [PubMed]

10. Wei, Z.C.; Li, X.P.; Zhong, N.F.; Tan, X.P.; Zhang, X.M.; Liu, H.Z.; Meng, H.Y.; Liang, R.S. Analogue electromagnetically induced transparency based on low-loss metamaterial and its application in nanosensor and slow-light device. Plasmonics 2016, 12, 641-647. [CrossRef]

11. Li, Z.Y.; Ma, Y.F.; Huang, R.; Singh, R.J.; Gu, J.Q.; Tian, Z.; Han, J.G.; Zhang, W.L. Manipulating the plasmon-induced transparency in terahertz metamaterials. Opt. Express 2011, 19, 8912-8919. [CrossRef]

12. Qiao, S.; Zhang, Y.X.; Xu, G.Q.; Sun, L.L.; Sun, H.; Li, L.; Liang, S.X.; Zhao, Y.C.; Yang, Z.Q. Controlling the transparency window in terahertz band using mode coupling metamaterials. J. Appl. Phys. 2015, 117, 063103. [CrossRef]

13. Zhang, H.Y.; Cao, Y.Y.; Liu, Y.Z.; Li, Y.; Zhang, Y.P. A novel graphene metamaterial design for tunable terahertz plasmon induced transparency by two bright mode coupling. Opt. Commun. 2017, 391, 9-15. [CrossRef]

14. Ding, G.W.; Liu, S.B.; Zhang, H.F.; Kong, X.K.; Li, H.M.; Li, B.X.; Liu, S.Y.; Li, H. Tunable electromagnetically induced transparency at terahertz frequencies in coupled graphene metamaterial. Chin. Phys. B. 2015, 24, 118103. [CrossRef]

15. Tian, S.C.; Wan, R.G.; Xing, E.B.; Rong, J.M.; Wu, H.; Wang, L.J.; Shu, S.L.; Tong, C.Z.; Ning, Y.Q. Tunneling induced transparency and giant Kerr nonlinearity in multiple quantum dot molecules. Physica E 2015, 69, 349-353. [CrossRef]

16. Xu, Q.; Su, X.Q.; Ouyang, C.M.; Xu, N.N.; Cao, W.; Zhang, Y.P.; Li, Q.; Hu, C.; Gu, J.Q.; Tian, Z.; et al. Frequency-agile electromagnetically induced transparency analogue in terahertz metamaterials. Opt. Lett. 2016, 41, 4562-4565. [CrossRef]

17. Khatua, S.; Chang, W.S.; Swanglap, P.; Olson, J.; Link, S. Active Modulation of Nanorod Plasmons. Nano Lett. 2011, 11, 3797-3802. [CrossRef]

18. Castro Neto, A.H.; Guinea, F.; Peres, N.M.R.; Novoselov, K.S.; Geim, A.K. The electronic properties of graphene. Rev. Mod. Phys. 2009, 81, 109-162. [CrossRef]

19. Li, X.M.; Li, T.; Chen, Z.F.; Fang, H.; Li, X.S.; Wang, X.R.; Xu, J.B.; Zhu, H.W. Graphene and related two-dimensional materials: Structure-property relationships for electronics and optoelectronics. Appl. Phys. Rev. 2017, 4, 021306. [CrossRef]

20. Liu, T.T.; Yi, Z.; Xiao, S.Y. Active control of near-field coupling in a terahertz metal-graphene metamaterial. IEEE Photonics Technol. Lett. 2017, 29, 1998-2001. [CrossRef]

21. Xiao, S.Y.; Wang, T.; Liu, T.; Yan, X.; Li, Z.; Xu, C. Active modulation of electromagnetically induced transparency analogue in terahertz hybrid metal-graphene metamaterials. Carbon 2018, 126, 271-278. [CrossRef]

22. Liu, T.T.; Wang, H.; Liu, Y.; Xiao, L.; Zhou, C.; Xu, C.; Xiao, S. Dynamically tunable electromagnetically induced transparency in a terahertz hybrid metamaterial. Physica E 2018, 104, 229-232. [CrossRef]

23. Zhao, X.L.; Yuan, C.; Lu, W.; Xu, S.; Yao, J.Q. Plasmon-Induced Transparency in Metamaterial Based on Graphene and Split-Ring Resonators. IEEE Photonics Technol. Lett. 2015, 27, 1321-1324. [CrossRef]

24. Chen, H.; Zhang, H.; Liu, M.; Zhao, Y.; Liu, S.; Zhang, Y. Tunable multiple plasmon-induced transparency in three-dimensional Dirac semimetal metamaterials. Opt. Commun. 2018, 423, 57-62. [CrossRef]

25. Cen, H.; Wang, F.; Liang, R. Tunable plasmon induced transparency based on bright-bright mode coupling graphene metamaterial. Opt. Commun. 2018, 420, 78-83. [CrossRef]

26. Liu, M.; Tian, Z.; Zhang, X.; Gu, J.; Ouyang, C.; Han, J.; Zhang, W.L. Tailoring the plasmon-induced transparency resonances in terahertz metamaterials. Opt. Express 2017, 25, 19844-19855. [CrossRef] 
27. Ordal, M.A.; Bell, R.J.; Alexander, R.W.; Long, L.L.; Querry, M.R. Optical properties of fourteen metals in the infrared and far infrared: $\mathrm{Al}, \mathrm{Co}, \mathrm{Cu}, \mathrm{Au}, \mathrm{Fe}, \mathrm{Pb}, \mathrm{Mo}, \mathrm{Ni}, \mathrm{Pd}, \mathrm{Pt}, \mathrm{Ag}, \mathrm{Ti}, \mathrm{V}$, and W. Appl. Opt. 1985, 24, 4493-4499. [CrossRef]

28. Zhang, J.; Guo, C.; Liu, K.; Zhu, Z.; Ye, W.; Yuan, X.; Qin, S. Coherent perfect absorption and transparency in a nanostructured graphene film. Opt. Express 2014, 22, 12524-12532. [CrossRef]

29. Ishikawa, A.; Tanaka, T. Plasmon hybridization in graphene metamaterials. Appl. Phys. Lett. 2013, 102, 253110. [CrossRef]

30. Jnawali, G.; Rao, Y.; Yan, H.; Heinz, T.F. Observation of a transient decrease in terahertz conductivity of single-layer graphene induced by ultrafast optical excitation. Nano Lett. 2013, 13, 524-530. [CrossRef]

31. Lee, S.H.; Choi, M.; Kim, T.T.; Lee, S.W.; Liu, M.; Yin, X.; Choi, H.K.; Lee, S.S.; Choi, C.G.; Choi, S.Y.; et al. Switching terahertz waves with gate-controlled active graphene metamaterials. Nat. Mater. 2012, 11, 936-941. [CrossRef]

32. Ye, Z.; Zhang, S.; Wang, Y.; Park, Y.S.; Zentgraf, T.; Bartal, G.; Yin, X.; Zhang, X. Mapping the near-field dynamics in plasmon-induced transparency. Phys. Rev. B 2012, 86, 155148. [CrossRef]

33. Hu, H.; Zhai, F.; Hu, D.; Li, Z.; Bai, B.; Yang, X.; Dai, Q. Broadly tunable graphene plasmons using an ion-gel top gate with low control voltage. Nanoscale 2015, 7, 19493-19500. [CrossRef]

34. Li, Q.; Cong, L.; Singh, R.; Xu, N.; Cao, W.; Zhang, X.; Tian, Z.; Du, L.; Han, J.; Zhang, W.L. Monolayer graphene sensing enabled by the strong Fano-resonant metasurface. Nanoscale 2016, 8, 17278-17284. [CrossRef]

35. Chiam, S.Y.; Singh, R.; Rockstuhl, C.; Lederer, F.; Zhang, W.L.; Bettiol, A.A. Analogue of electromagnetically induced transparency in a terahertz metamaterial. Phys. Rev. B 2009, 80, 153103. [CrossRef]

36. Gu, J.; Singh, R.; Liu, X.; Zhang, X.; Ma, Y.; Zhang, S.; Maier, S.A.; Tian, Z.; Azad, A.K.; Chen, H.-T.; et al. Active control of electromagnetically induced transparency analogue in terahertz metamaterials. Nat. Commun. 2012, 3, 1151. [CrossRef]

37. Yin, X.; Feng, T.; Yip, S.; Liang, Z.; Hui, A.; Ho, J.C.; Li, J. Tailoring electromagnetically induced transparency for terahertz metamaterials: From diatomic to triatomic structural molecules. Appl. Phys. Lett. 2013, 103, 021115. [CrossRef] 